\title{
RECURSOS FISIOTERAPÊUTICOS UTILIZADOS NO TRATAMENTO DE INCONTINÊNCIA URINÁRIA: REVISÃO DE LITERATURA
}

\author{
Silvia Carolinne Pereira RIBEIRO ${ }^{1}$ \\ Wendel Nei Teles BRITO' \\ Ramon Reis NAST ${ }^{1}$ \\ Flavia Maria Lessa MELO² \\ Rafaela Cordeiro de MACÊDO ${ }^{3}$
}

\begin{abstract}
${ }^{1}$ Graduandos do Curso de Fisioterapia do Centro Universitário do Pará (CESUPA). silviaribeirocp@gmail.com, wendel_a7x@hotmail.com, ramonreisnast@gmail.com ${ }^{2}$ Fisioterapeuta. Mestre em Saúde, Sociedade e Endemias na Amazônia. Docente do Centro Universitário do Pará. flaviamlmelo@yahoo.com.br

${ }^{3}$ Fisioterapeuta. Mestranda em Ensino e Saúde da Amazônia. Docente do Centro Universitário do Pará (CESUPA) rafaela_cmacedo@hotmail.com
\end{abstract}

\section{Recebido em: 10/01/2011 - Aprovado em: 08/02/2016 - Disponibilizado em: 30/07/2016}

RESUMO: O presente estudo trata-se de uma revisão de literatura, onde objetivou-se buscar evidências sobre os recursos fisioterapêuticos utilizados no tratamento de incontinência urinária.Trata-se de um estudo considerado descritivo e comparativo, onde foi realizado levantamento de artigos científicos, publicados entre 2005 à 2015 . A pesquisa foi realizada entre os meses de agosto a dezembro de 2015. Foram utilizados para a busca dos artigos as bases de referências bibliográficas: Medical LiteratureAnalysisandRetrieval System Online - MEDLINE (PUBMED), Literatura Latino-Americana e do Caribe em Ciências da Saúde (LILACS) e ScientificElectronic Library Online (Scielo). Como descritores de assunto foram utilizadas as palavras: "fisioterapia uroginecológica", "incontinência urinária", "assoalho pélvico", "eletroestimulação".O presente estudo contou com uma amostra final de 21 artigos após aplicação dos critérios de inclusão e exclusão. De modo geral, o tipo de Incontinência Urinária que recebeu mais tratamento foi a de Esforço $(66,66 \%)$, sendo mais frequente utilizar terapias combinadas para realizar o tratamento $(33,33 \%)$, a amostra variou entre 1 a 80 pacientes e foi encontrada melhora significante em 19 artigos (76\%). O número de sessões variou de acordo com o tipo de recurso utilizando, sendo que a Ginástica Hipopressiva foi o que obteve melhor resultado relacionado ao número de sessões.Diante do exposto confirmou-se a eficácia da atuação fisioterapêutica com diferentes recursos, com exceção do uso de orientações como única forma de tratamento, que não obteve resultados significantes.

PALAVRAS-CHAVE: Urologia. Ginecologia. Fisioterapia. IncontinênciaUrinária. AssoalhoPélvico.

\section{PHYSIOTHERAPEUTIC RESOURCES UTILIZED IN THE TREATMENT OF URINARY INCONTINENCE: REVISION OF LITERATURE}

\begin{abstract}
The present study is a revision of literature, where the objective was to search for evidence on the physiotherapeutic resources utilized in the treatment of urinary incontinence. It is a descriptive and comparative study, where scientific articles were used, published between 2005 through 2015. The research was done between the months of August through December of 2015. The following bibliographical reference databases were used for the search of articles: Medical LiteratureAnalysisandRetrieval System Online - MEDLINE (PUBMED), Literatura LatinoAmericana e do Caribe emCiências da Saúde (LILACS) e ScientificElectronic Library Online (Scielo). As subject descriptors the following words were used: urinary, incontinence, physical therapy urological, physical therapy gynecological. The study at hand counted with a sample of 21 articles after the criteria for inclusion and exclusion were applied. As a general, the type of Urinary Incontinence that receive the most treatment was the Effort type (66.66\%), with the more frequent choice being to make use of combined therapies to handle treatment (33.33\%), the sample varied between 1 and 80 patients and significant improvement was found in 19 of the articles (76\%). The number of sessions varied according to the type of resource used, with Hipopresiva Gymnastics being the one that obtained the best result in relation to the number of sessions. In the exposure, the efficacy of using different resources in the physiotherapeutic action was confirmed, with the exception of the use of orientations as the only form of treatment, which did not receive significant results.
\end{abstract}

KEYWORDS: Urological. Gynecological. Physical Therapy. UrinaryIncontinence. PelvicFloor. 


\section{INTRODUÇÃO}

No contexto de saúde, a fisioterapia uroginecológica é de suma importância para a prevenção de disfunções do assoalho pélvico (LEITE \& POÇAS, 2015). Atualmente a patologia de maior incidência dentro da fisioterapia uroginecológica é a Incontinência Urinária (IU)(TAVARES et al, 2011; GAMEIRO, 2015) afetando grande parte da população mundial e, de acordo com a Sociedade Brasileira de Urologia,um a cada 25 indivíduos poderá vir a desenvolver incontinência urinária e tem incidência de cerca de $40 \%$ em mulheres pós-menopausa e $5 \%$ em homens pós-prostectomizados(SILVA et al, 2015).

A Incontinência Urinária é definida pela perda de urina, de forma involuntária, podendo ser de três tipos: Incontinência Urinária de Esforço (IUE), Incontinência Urinária de Urgência (IUU) ou mista (IUM)(RETT et al, 2015; MOURÃO et al, 2015). Sendo a IUE, o tipo mais comum de IU, tem como queixa principal a perda involuntária de urina decorrente de algum esforço, espirro ou tosse, pelo fato de estes desencadearem um aumento da pressão intraabdominal (MOURÃO et al, 2015; STEPHENSON \& O' CONNOR, 2014; HIGA, LOPES \& REIS, 2015). A IUU apresenta-se também pela perda de urina, porém com uma intensa vontade de urinar, podendo estar presente a não-inibição da atividade do músculo detrusor. A IUM é a combinação dos dois tipos de IU acima citadas, sendo esta a menos freqüente (RETT et al, 2015; MOURÃO et al, 2015).

Como fatores de risco para a IU encaixam-se: idade, sendo esta considerada um dos principais, pela diminuição da capacidade de bexiga, caindo de 500 a $600 \mathrm{ml}$ para 250 a 300ml, além dos baixos níveis de estrogênio, doenças crônicas e aumento do índice de massa corpórea (IMC); a obesidade, sendo apontada pelo aumento da pressão intra-abdominal e consequentementeaumento da pressão intravesical, alterando, assim, o mecanismo do trato urinário; a paridade; os tipos de parto, tanto normal e cesárea; uso de anestesia durante o parto, por provocar prolongamento do segundo estágio do trabalho de parto, aumentando, como consequência, os riscos de lesão do assoalho pélvico (AP); peso do recém-nascido, pelo aumento da pressão intra-abdominal, em conjunto com o risco de lesão do AP, principalmente em crianças com peso maior de 4000g; menopausa, pelo fato da estática pélvica sofrer alterações com as mudanças hormonais, além do fato do hipoestrogenismo predispor a mulher ao aumento da frequência e urgência; exercícios físicos rigorosos; constipação intestinal; fatores hereditários; doenças crônicas; uso de drogas lícitas e ilícitas e consumo de cafeína (BEUTTENMÜLLER et al, 2015) 
As disfunções urinárias, como um todo, são de estrema relevância para a Área da Saúde por se tratarem de patologias que geram bastante constrangimento, apresentando graves complicações, sendo estas de caráter social, ocupacionais, psicológicas, físicas (de saúde), sexuais e/ou econômicas (STEPHENSON \& O' CONNOR, 2004), interferindo negativamente na qualidade de vida do indivíduo, não sendo exclusivo de mulheres idosas, podendo ocorrer também em mulheres jovens(HIGA, LOPES, \& REIS, 2015; BERZELI, MARTINS \& DREHER, 2013; LOPES \& HIGA, 2015)

Este estudo propõe apresentar uma revisão de literatura buscando evidências sobre os recursos fisioterapêuticos utilizados no tratamento de incontinência urinária.

\section{METODOLOGIA}

Trata-se de um estudo descritivo e comparativo, onde foi realizado levantamento de artigos científicos, de caráter experimental, publicados entre 2005 à 2015. A pesquisa foi realizada entre os meses de agosto a dezembro de 2015. Os artigos apresentavam dois idiomas: português e inglês. Foram utilizadas para a busca dos artigos as bases de referências bibliográficas: Medical LiteratureAnalysisandRetrieval System Online - MEDLINE (PUBMED), Literatura Latino-Americana e do Caribe em Ciências da Saúde (LILACS) e ScientificElectronic
Library Online (Scielo). Como descritores de assunto foram utilizadas as palavras: fisioterapia uroginecológica, incontinência urinária, assoalho pélvico, eletroestimulação (em português), "urinary”, “incontinence, physicaltherapyurological", physical therapy gynecological” (em inglês). Foram excluídos artigos de revisões de literatura ou de perfil epidemiológico, que os indivíduos do estudo tivessem outra patologia ou distúrbio associado, estudos que não especifiquem o protocolo de atendimento ou de tratamentos cirúrgicos.

\section{RESULTADOS}

O estudo teria uma amostra total de 266 artigos, porém após aplicação dos critérios de inclusão e exclusão da pesquisa, a amostra final foi de 21 artigos.

Pelos artigos selecionados, 14realizaram tratamento de IUE $(66,66 \%), 1$ de IUM (4,76\%), nenhum falou apenas de IUU, 3 não especificaram qual o tipo de IU a ser tratada $(14,28 \%)$, sendo que, dentre os artigos, 3 trataram mais de um tipo de IU $(14,28 \%)$ como descrito na tabela 01 .

Tabela 01. Tipos de Incontinência Urinária abordados nos artigos.

\begin{tabular}{ccc}
\hline Tipos de IU & N=21 & $\mathbf{1 0 0 \%}$ \\
\hline IUE & 14 & 66,66 \\
IUM & 1 & 4,76 \\
Mais de um tipo de IU & 3 & 14,28 \\
Não Especificado & 3 & 14,28 \\
\hline Fonte: Levantamento bibliográfico, 2015.
\end{tabular}


Foram utilizados tratamentos com um único recurso e com terapias combinadas, onde, em alguns artigos, foram utilizados recursos diferentes para fazer comparações. A cinesioterapia foi utilizada em 3 artigos $(12,5 \%)$. A eletroterapia foi utilizada como o único recurso também em 3 artigos (12,5\%). O recurso Ginástica Hipopressiva $(\mathrm{GH})$ foi utilizado em 2 artigos (8,33\%). As técnicas de Reeducação Postural Global (RPG) foram utilizadas em apenas 1 artigo dentre os selecionados $(4,16 \%)$. O Treinamento da Musculatura do Assoalho Pélvico (TMAP) foi utilizado em 2 artigos (8,33\%). Os cones vaginais foram utilizados também em 2 artigos $(8,33 \%)$. O pilates foi utilizado como forma de tratamento da incontinência urinária em 3 artigos (12,5\%). Em 8 artigos (33,33\%) foram utilizados recursos em conjunto, sendo a cinesioterapia como recurso primário, seguido de eletroterapia e ginástica hipopressiva, como demonstrado na tabela 2.

Tabela 02. Recursos utilizados

\begin{tabular}{ccc}
\hline Recurso & $\mathbf{N = 2 4}$ & \% \\
\hline Cinesioterapia & 3 & 12,5 \\
Eletroterapia & 3 & 12,5 \\
Ginástica Hipopressiva & 2 & 8,33 \\
Reeducação Postural Global & 1 & 4,16 \\
Treinamento da musculatura do & 2 & 8,33 \\
assoalho pélvico & & \\
Cones Vaginais & 2 & 8,33 \\
Pilates & 3 & 12,5 \\
\hline Recursos utilizados em conjunto & 8 & 33,33 \\
\hline Fonte: Levantamento bibliografico, & 2015. &
\end{tabular}

Fonte: Levantamento bibliográfico, 2015.

$\mathrm{O}$ número de pacientes na literatura selecionada variou entre 1 paciente, em estudos que utilizaram como metodologia relato de caso, a 80 pacientes, onde 10 artigos $(47,61 \%)$ tiveram uma amostra de 1 a 10 pacientes, 2 contaram com uma amostra de 11 a 20 pacientes $(9,5 \%), 5$ com uma amostra de 21 a 30 pacientes $(23,7 \%), 3(15 \%)$ com a amostra de 31 a 40 pacientes e apenas 1 $(4,76 \%)$ com uma amostra maior que 40 pacientes.

Gráfico 01.Amostra utilizada nos artigos

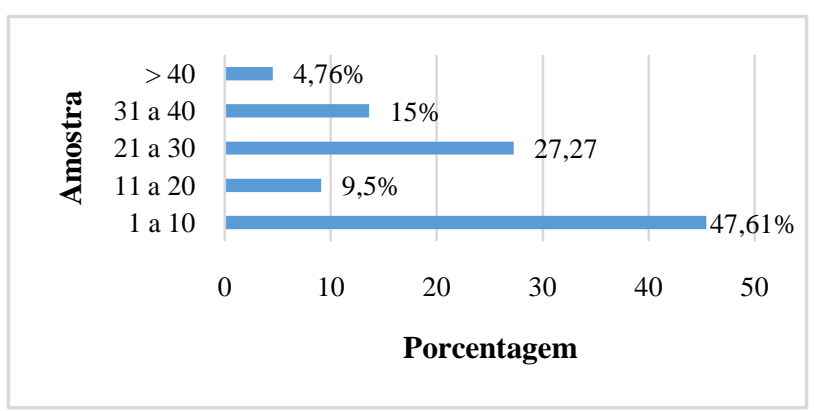

Fonte: Levantamento bibliográfico, 2015

Relacionando aos métodos avaliativos utilizados,alguns estudos tiveram mais de um tipo de resultado encontrado, também pelo fato dos autores utilizarem mais de um tipo de recurso. A melhora significante foi o resultado mais encontrado, estando presente em 19 artigos (76\%), seguindo por 3 artigos que não houve resultado significante (12\%), a cura, ou resolução do problema, foi encontrada como resultado em 2 estudos (8\%) e discreta melhora em apenas 1 (4\%), como mostra o gráfico 02 . 
Gráfico 02. Resultados dos tratamentos relatados nos artigos

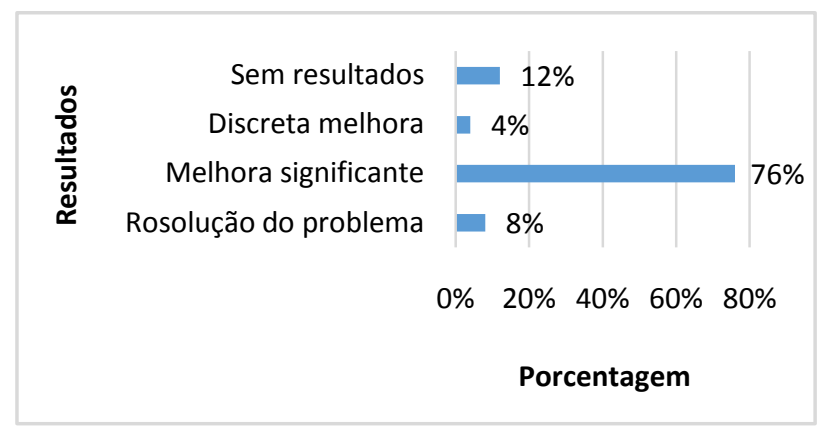

Fonte: Levantamento bibliográfico, 2015

\section{DISCUSSÃO}

A IU destaca-se como um problema de saúde, atingindo indivíduos de todas as faixas etárias e de ambos os sexos, gerando comprometimentos físicos, sociais, ocupacionais, psicológicas, sexuais e econômicas, interferindo negativamente em sua qualidade de vida, sendo de extrema relevância para a área da saúde, como um todo (TAVARES, 2011; STEPHENSON, 2004; LOPES \& HIGA, 2015; BERZELI, MARTINS \& DREHER, 2013). A fisioterapia é indicada pela Sociedade Internacional de Continência como opção de primeira linha para o tratamento de IU por seu baixo custo e comprovada eficácia (FIGUEREIDO, 2015).

Um dos recursos utilizados nas pesquisas foi a cinesioterapia. Esta aplicada à área da fisioterapia uroginecológica objetiva a obtenção de maior propriocepção e fortalecimento muscular dos MAP, utilizando como recursos a bola suíça e bastões, baseando-se em técnicas como o contrair manter - relaxar associados a exercícios ativos livres a fim de proporcionar um melhor controle urinário (VALÉRIO, CARVALHO \&SILVA, 2013; GOMES et al, 2009; OLIVEIRA, 2006). Já o treino da musculatura do assoalho pélvico (TMAP) consiste no mesmo princípio da terapia anteriormente citada, porém especificamente à esta musculatura, podendo ser realizado com dispositivos auxiliares como cones vaginais, que possuem diferentes pesos variando de $20 \mathrm{e}$ 100gpara resistência dos MAP por meio de estímulos, onde a paciente deve retê-los internamente (MATHEUS et al, 2006; SANTOS et al, 2009). Nos artigos que citaram estes recursos como forma de tratamento foram realizadas entre 4 a 36 sessões ou 2 a 24 semanas, sendo que $100 \%$ destes apresentaram melhora significativa, não havendo relação entre o número de sessões e semanas com os resultados encontrados.

Outro método de tratamento citado foi a eletroestimulação, que é utilizada como forma de tratamento por gerar impulsos de baixa intensidade, assim estimulando a contração muscular individualizada nos MAP, agindo ainda sem o auxílio dos pacientes que possuem menor consciência da região pélvica, ou mesmo atuando em conjunto no auxílio dos pacientes com uma maior percepção, sendo eficaz também com o objetivo de melhorar propriocepção. Esse recurso é utilizado por via de um eletrodo em formato anatômico, com possibilidade de ser realizado por via vaginal ou anal, sendo necessário um 
eletrodo específico para cada uma destas e sua principal função é a obtenção do controle e ganho de força dos MAP, inclusive na hiperatividade do músculo detrusor da bexiga (BEUTTENMÜLLER et al, 2011; SANTOS et al, 2009). Os métodos de tratamento com eletroestimulação utilizaram biofeedback e Dualpex ${ }^{\circledR}$, com número de sessões entre 5 a 32 ou 2 a 16 semanas, apresentando melhoras significativas em $100 \%$ destes, também não apresentando relação direta com o número de sessões ou semanas.

Outro método utilizado pelos autores é a Ginástica Hipopressiva, que é uma combinação de exercícios da musculatura abdominal, do assoalho pélvico e peitoral, sendo realizados em três fases: inspiração diafragmática lenta e profunda; expiração completa e aspiração diafragmática. Tem como objetivo elevar tanto os órgãos abdominais quanto MAP e sua diferença em relação a outros exercícios com o mesmo objetivo é que este não necessita que o paciente tenha domínio proprioceptivo dos MAP, tendo uma atuação significativamente importante para prevenção e tratamento de distúrbios uroginecológicos, dentre eles a IU (COSTA et al, 2011).Os artigos que utilizaram este método obtiveram discreta melhora com 5 sessões, duas vezes por semana, melhora significativa de 4 ou 36 sessões sendo estas uma ou três vezes na semana, e resolução do problema com 3 sessões em 12 semanas, apesar de ser realizada apenas uma vez na semana, demonstrando a eficácia do método.

A técnica da Reeducação Postural Global (RPG) tem sua importância partindo do conhecimento de que, os MAP tem importante participação na manutenção da estabilidade lombar, sendo a instabilidade da mesma grande geradora de pressão e fadiga desses músculos, com isso causando stress contínuo e consequentemente, fraqueza (FOZZATTI et al, 2008). A prática de Pilates também possui resultados importantes na IU, seus principais pilares são a reeducação postural fortalecimento da musculatura pélvica, dando importância à respiração e controle abdominal, realizando principalmente contrações concêntricas que atuam principalmente na musculatura estática, podendo prevenir ou tratar a IU (STELTER \& FRARE, 2014). O tempo de tratamento dos artigos que utilizaram como técnica o RPG variou de 18 a 36 sessões ou 12 a 24 semanas e os que utilizaram o pilates como método de tratamento realizaram de 16 a 24 sessões e 8 a12 semanas, sendo que ambos os métodos apresentaram $\quad 100 \%$ de melhoras significativas.

Enquanto que as orientações, realizadas com o objetivo de um trabalho contínuo, tendo em vista que as sessões por si só muitas vezes não alcançam a cura total, onde é sempre necessária a colaboração do paciente para seu próprio bem estar, sendo 
estes orientados acerca dos exercícios a serem realizados, a postura que deve adotar no dia a dia e a ingestão de líquidos para regularizar a micção (MARQUES \& FREITAS, 2005). O método de tratamento somente por orientações não se mostrou muito eficaz nos artigos, não havendo como relacionar com o número de sessões ou semanas, tendo em vista que foram realizadas em domicílio, sem a presença física do terapeuta, e é possível que esta seja a causa dos estudos que utilizaram este método como única forma de tratamento não apresentar melhora dos sintomas de IU. Contudo, quando realizadas orientações simultaneamente a outros recursos, como cinesioterapia e cones, as orientações apresentam-se eficazes.

De acordo com Matheus et al (2006) pode-se afirmar que metodologias utilizando terapias combinadas apresentam um melhor nível de eficácia na resolução da queixa ou diminuição dos sintomas dos quadros de IU.

\section{CONCLUSÃO}

Diante do exposto confirmou-se a eficácia da atuação fisioterapêutica no tratamento das diferentes formas de IU, apresentando resultados expressivos tanto no fortalecimento da MAP, quanto na propriocepção da mesma. Verificou-se que a Ginástica Hipopressiva apresenta melhores resultados em relação aotempo de aplicação sendo aplicada como única forma de tratamento ou, ainda, associada às demais técnicas. Além disso, todas as técnicas citadas nos estudos apresentaram-se eficazes, com exceção dos estudos que utilizaram como única forma de tratamento as orientações para serem realizados exercícios em domicílio, mostrando que estas devem ser associadas com outras técnicas para garantir melhora do quadro de IU.

\section{REFERÊNCIAS}

BERZELI, E. M., MARTINS, M., DREHER, D. Z. Programa individualizado de exercícios para incontinência urinária executado no espaço domiciliar.Scientia Medica (Porto Alegre) 2013; volume 23, número 4, p. 232238

BEUTTENMÜLLER, L. et al. Contração muscular do assoalho pélvico de mulheres com incontinência urinária de esforço submetidas a exercícios e eletroterapia: um estudo randomizado.Fisioter. Pesqui. São Paulo, vol. 18, n.3, Jul/Set. 2011. Disponível em:

<http://www.scielo.br/pdf/fp/v18n3/02.pdf>. Acesso em: 06 Ago. 2015.

COSTA et al. Ginástica hipopressiva como recurso proprioceptivo para os músculos do assoalho pélvico de mulheres incontinentes.

Rev. Fisioterapia Brasil, v. 12, n. 5, set/out, 2011. Disponível em: $<$ http://abafi.com.br/downloads/ginasticahipopressiva-como-recursoproprioceptivo.pdf>. Acesso em: 23 Nov. 2015.

FIGUEIREDO et al. Perfil epidemiológico e clínico de usuárias de serviços de fisioterapia uroginecológica da rede pública.

RevBrasFisioter, 12(2):136-42, MG, 2008. Disponível em: http://www.scielo.br/pdf/rbfis/v12n2/a10v12n 2.pdf $>$. Acesso em: 30 Nov. 2015. 
FOZZATTI et al. Impacto na reeducação postural global no tratamento da incontinência urinária de esforço feminina.

RevAssocMedBras, v. 54. n. 1, p. 17-22, 2008 Disponível em:

<http://www.scielo.br/pdf/ramb/v54n1/15.pdf >. Acesso em: 01 Dez. 2015.

GAMEIRO, L. F. O. Estimulação elétrica com eletrodos não implantáveis no tratamento da bexiga hiperativa em adultos: revisão sistemática de ensaios clínicos randonizados. 2014. $233 \mathrm{f}$. Tese (doutorado) - Universidade Estadual Paulista Júlio de Mesquita Filho, Faculdade de Medicina de Botucatu, 2014. Disponível em: <http://hdl.handle.net/11449/123785>. Acesso em: 06 Ago. 2015.

GOMES et al. Efeito da cinesioterapia e eletroestimulação transvaginal na incontinência urinária feminina: estudo de caso. Rev. ArqCiênc Saúde, v. 6, n. 2, p. 8388, abr/jun, 2009. Disponível em:

$<$ http://repositorio-racs.famerp.br/racs_ol/vol16-2/ID326.pdf>. Acesso em: 02 Dez. 2015.

HIGA, R., LOPES, M. H. B. M., REIS, M. J. Fatores de risco para incontinência urinária na mulher. RevEscEnferm, USP, 2008;

42(1):187-92. Disponível em: <http://www.scielo.br/pdf/reeusp/v42n1/25.pd f>. Acesso em: 25 nov. 2015.

LEITE, J.; POÇAS, F. Tratamento da incontinência fecal. Rev Port Coloproct, v. 7, n. 2, p. 68-72, 2010. Disponível em: <http://www.spcoloprocto.org/uploads/recom endac807_o771_es_tratamento_da_incontine 770_ncia_fecal.pdf>. Acesso em: 06 Ago. 2015.

LOPES, M. H. B. M., HIGA, R. Restrições causadas pela incontinência urinária à vida da mulher. Rev. Esc. Enferm, 2008; 40(1); 3441. Disponível em:

<http://www.scielo.br/scielo.php?pid=S0080$62342006000100005 \&$ script=sci_arttext $>$. Acesso em: 25 Nov. 2015.
MARQUES, S.M.F. \& FREITAS, P.A.C. Acinesioterapiacomo tratamento da incontinênciaurinária na unidade básica de saúde. Rev.Fisioterapia em Movimento. v.18, n.4, pp.63-67,2005. Disponível em: <http://www2.pucpr.br/reol/public/7/archive/ 0007-00000232-

A_CINESIOT\%5B1\%5D.....PDF>. Acesso em: 22 Nov. 2015.

MATHEUS et al,influência dos exercícios perineais e dos cones vaginais, associados à correção postural, no tratamento da incontinência urinária feminina. Rev. bras.

fisioter.,, v. 10, n. 4, p. 387-392, out./dez, São Carlos, 2006. Disponível em: <http://www.scielo.br/pdf/rbfis/v10n4/04.pdf >. Acesso em: 21 Out. 2015.

MOURÃO et al. Prevalência de queixas urinárias e o impacto destas na qualidade de vida de mulheres integrantes de grupos de atividade física. Rev. Acta Fisiátr. 2008;15(3):170-175. Disponível em: $<$ http://www.actafisiatrica.org.br/detalhe artigo.asp?id=139.. Acesso em: 21 Nov. 2015.

OLIVEIRA, C. Efeitos da cinesioterapia no assoalho pélvico durante o ciclo gravídicopuerperal. 2006. 99f. Tese (Mestrado em Ciências) - Faculdade de Medicina, Universidade de São Paulo, São Paulo. 2006. Disponível em: <http://www.feminafisio.com/noticias/10003/ livro_10003.pdf>. Acesso em: 02 Dez. 2015.

RETT, M. T. et al. Qualidade de vida em mulheres após tratamento da incontinência urinária de esforço com fisioterapia. Rev. Bras. Ginecol. Obstet., Rio de Janeiro, v.29, n.3, Mar. 2007. Disponível em: <http://www.scielo.br/pdf/rbgo/v29n3/04.pdf $>$. Acesso em: 26 Set. 2015.

SANTOS, P. F. D. et al. Eletroestimulação funcional do assoalho pélvico versus terapia com os cones vaginais para o tratamento de incontinência urinária de esforço. Rev. Bras. Ginecol. Obstet., Rio de Janeiro, v.31, n.9, Set. 2009 . Disponível em: 
<http://www.scielo.br/pdf/rbgo/v31n9/a05v31

n9.pdf $>$. Acesso em: 06 Ago. 2015

SILVA, J. S et al. Repercussão da

incontinência urinária na qualidade de vida de mulheres idosas institucionalizadas.

Suplemento Especial, v.13, n. 6, Nov./Dez.

2012. Disponível em:

$<$ http://assoalhopel.dominiotemporario.com/d oc/Fisioterapia_Brasil_v13n6_Supl_Saude_da _Mulher_fim.pdf>. Acesso em: 06 Ago. 2015.

STELTER, S. S., FRARE, J. C. Influência do método pilates no tratamento e na qualidade de vida de mulheres com incontinência urinária. FIEP BULLETIN, v. 8, 2014. Disponível em: < http://www.fiepbulletin.net/index.php/fiepbull etin/article/view/4583>. Acesso em: 01 Dez. 2015.

STEPHENSON, R. G.; O' CONNOR, L. J. Fisioterapia aplicada à ginecologia $\mathrm{e}$ obstetrícia. 2. ed. Barueri: Manole, 2004.

TAVARES et al. Qualidade de vida de idosos com incontinência urinária. Rev. Eletr. Enf. out/dez;13(4), pag. 695-702, 2011.

VALÉRIO, T. M. O. S., CARVALHO, J. A., SILVA, E. B. Cinesioterapia na incontinência urinária de esforço da mulher. Revista

Científica do ITPAC, v.6, n. 4 Araguaína,2013. Disponível em: <http://www.itpac.br/arquivos/Revista/64/7.p df $>$. Acesso em: 01 Dez. 2015. 